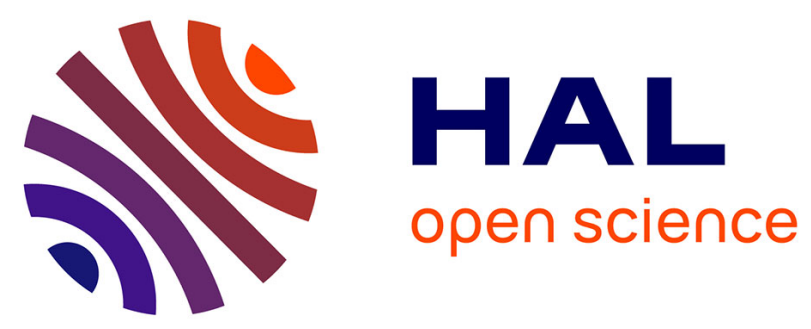

\title{
Politique du couple: amour réciproque et légitimité dynastique dans Britannicus
}

\author{
Volker Schröder
}

\section{To cite this version:}

Volker Schröder. Politique du couple: amour réciproque et légitimité dynastique dans Britannicus. Cahiers de l'Association internationale des études françaises (CAIEF), 1997, 49 (1), pp.455-491. 10.3406/caief.1997.1299 . halshs-02299709

\section{HAL Id: halshs-02299709 \\ https://shs.hal.science/halshs-02299709}

Submitted on 27 Sep 2019

HAL is a multi-disciplinary open access archive for the deposit and dissemination of scientific research documents, whether they are published or not. The documents may come from teaching and research institutions in France or abroad, or from public or private research centers.
L'archive ouverte pluridisciplinaire HAL, est destinée au dépôt et à la diffusion de documents scientifiques de niveau recherche, publiés ou non, émanant des établissements d'enseignement et de recherche français ou étrangers, des laboratoires publics ou privés.

\section{(1)(1) $\$(0)$}

Distributed under a Creative Commons Attribution - NonCommercial - ShareAlikel 4.0 


\section{Politique du couple : amour réciproque et légitimité dynastique dans}

\section{Britannicus}

\section{Volker Schroder}

\section{Citer ce document / Cite this document :}

Schroder Volker. Politique du couple : amour réciproque et légitimité dynastique dans Britannicus. In: Cahiers de I'Association internationale des études francaises, 1997, n49. pp. 455-491;

doi : https://doi.org/10.3406/caief.1997.1299

https://www.persee.fr/doc/caief_0571-5865_1997_num_49_1_1299

Fichier pdf généré le 21/04/2018 
Nous publions ci-dessous le texte de l'article qui a mérité le Prix annuel de l'Association, réservé à un jeune chercheur. Le lauréat, Volker Schröder, enseigne à l'université de Salzburg. Son article, que nous reprenons avec l'aimable autorisation des éditeurs, a paru pour la première fois dans Politica e letteratura in Francia nel Cinquecento e nel Seicento, Paris-Bari, Nizet-Adriatica, 1997 (N.D.L.R.).

\section{POLITIQUE DU COUPLE : AMOUR RÉCIPROQUE ET LÉGITIMITÉ DYNASTIQUE DANS BRITANNICUS}

Le hasard qui fait qu'au programme de notre colloque sur " Politique et littérature " figure le nom de Racine, et non pas celui de Corneille, me semble symptomatique d'une certaine évolution qui est en train de modifier peu à peu l'image des deux grands dramaturges. Si Corneille demeure certes le point de référence obligé pour définir la formule de la «tragédie politique » (1), des critiques de plus en plus nombreux s'emploient à rectifier le cliché invétéré d'un Racine " apolitique ». Les travaux qui étudient dans le théâtre racinien des thèmes politiques l'image du souverain et de la cour, le rôle des conseillers, la présence du machiavélisme... - se sont multipliés ; souvent, on découvre chez Racine même une véritable " méditation » sur l'essence du " pouvoir », une "pensée politique » en relation plus ou moins étroite avec l'absolu-

(1) A titre d'exemple, comparer les intitulés des volumes respectifs de la collection "Que sais-je?" : Corneille et la tragédie politique de Georges Couton, Racine et la tragédie classique d'Alain Niderst. Ce couple d'étiquettes ne suggère-t-il pas que la tragédie " classique " ne serait pas " politique" ? 
tisme de Louis XIV (2). Cependant, comme l'a noté récemment Jean Rohou, la politique demeure "un thème d'importance controversée » (3), que beaucoup de critiques continuent de considérer, à la suite de Paul Bénichou, comme un simple "prétexte", un " ornement, plus ou moins important, d'une action plus substantielle » (4).

Dans ma communication, je voudrais aborder cette problématique à partir d'un aspect restreint, en examinant une catégorie de personnages rarement étudiée dans une perspective politique : les couples d'amoureux qui s'aiment d'un amour réciproque. D'Antigone et Hémon à Aricie et Hippolyte, en passant notamment par Junie et Britannicus, Atalide et Bajazet, Monime et Xipharès, l'amour de ces couples tendres, nobles et purs, innocents et malheureux, poétiques et pathétiques, contraste avec toutes les amours-passions non partagées dont fourmille le théâtre de Racine. Longtemps, on s'est contenté de critiquer ces "parfaits amants" comme des personnages fades, dépourvus de grandeur tragique, qui ne s'expliqueraient que par la concession regrettable que le dramaturge aurait été obligé de faire aux conventions du genre et au goût "précieux » ou "galant » du public féminin. Plus récemment, les critiques ont mieux rendu justice à ces personnages en les rattachant à la tradition romanesque et pastorale (5). Dans cette perspective, les couples d'amants

(2) Ne pouvant énumérer ici toutes ces études, je renvoie au bilan qu'en a dressé Ronald W. Tobin : "And therein lies the major innovation of recent Racine criticism : in all of these books and articles Racine is seen as engaging in an implicit dialogue with the crown. This supposes that his political discourse is informed, and so for the first time the heretofore unutterable has been uttered: "Racine penseur " ("Molière and Racine, or the Red and the Black" , [in] Actes de Columbus, éd. Charles G.S. Williams, Paris-Seattle-Tübingen : PFSCL, 1990 [Biblio 17, vol. 59], pp. 13-14).

(3) Jean Rohou, Jean Racine : bilan critique, Paris, Nathan, 1994, p. 53.

(4) Paul Bénichou, Morales du grand siècle, Paris, Gallimard, 1948 (rééd. 1967, coll. "idées"), p. 247.

(5) Voir surtout Marie-Odile Sweetser, "Présence du romanesque che» Racine : le couple amoureux dans La Thébaïde et Alexandre ", Travaux de littérature I (1988), pp. 91-104 ; Jacques Morel, Racine en toute's lettres, Paris, Bordas, 1992, pp. 59-60 ; Christian Delmas, La Tragédie de l'âge classique, Paris, Seuil, 1994, pp. 228-229. 
représentent une idylle amoureuse écrasée par le monde cruel du pouvoir; dénués d'ambition, n'aspirant qu'au bonheur "privé " de leur amour, ils ne seraient que les victimes passives d'une lutte politique menée par d'autres.

A y regarder de plus près, cependant, on découvre que les couples d'amoureux entretiennent un rapport à la fois plus étroit et plus ambigu avec la politique, qui ne leur est pas aussi étrangère qu'on pourrait le croire de prime abord. Je voudrais essayer de montrer comment une telle dimension politique, présente dans le texte racinien de façon discrète mais précise, contribue à la construction de ces personnages, et par là à celle du sens de la tragédie. Dans le cadre de cette communication, je suis obligé de me borner à l'étude de Britannicus, pour dégager un schéma dont on constatera les variations dans d'autres pièces, à première vue moins « politiques », de Racine.

\section{Les origines de Junie : Racine entre Tacite et Sénèque}

On sait que le couple d'amants de Britannicus doit son existence à l'invention d'un personnage féminin ajouté par Racine aux données historiques qui fournissent le sujet de sa tragédie. La création de Junie (comme plus tard celle de ses homologues Atalide, Monime et Aricie) permet le développement d'une intrigue sentimentale : du moment que Néron tombe amoureux de Junie, une rivalité passionnelle se greffe sur sa rivalité politique avec Britannicus. Ainsi, la fonction de Junie semble résider tout entière dans son rôle d'amante malheureuse violée par le tyran : "Donner à Britannicus une amoureuse, c'était créer un couple de victimes [...] dont les amours contrariées pouvaient donner à la pièce une dimension élégiaque de nature à contrebalancer la tonalité fortement politique de la donnée initiale " (6).

(6) Ceorges Forestier, préface à son édition de Britannicus, Paris, Gallimard (Folio théâtre), 1995, p. 14. 
Mais les nécessités de l'intrigue amoureuse ne suffisent pas à expliquer pourquoi Junie est " de la famille d'Auguste " (comme Atalide est " fille du sang ottoman », et Aricie "princesse du sang royal d'Athènes» (7)). En revanche, l'appartenance des amoureuses raciniennes à des familles non seulement nobles, mais princières revêt une importance politique assez précise dès lors que l'on considère la tragédie racinienne comme une tragédie dynastique. Il convient alors de suivre de plus près la piste des indications généalogiques données dans le texte, pour essayer de reconstruire la constellation dynastique qui sous-tend l'action politique et en détermine le sens.

En fait, comme le souligne Racine dans ses deux préfaces, Junie est un personnage non pas inventé de toutes pièces, mais modelé sur une figure historique, peu connue mais attestée par Sénèque, Tacite et Suétone (8) :

Junie ne manque pas non plus de censeurs. Ils disent que d'une vieille coquette, nommée Junia Silana, j'en ai fait une jeune fille très sage. Qu'auraient-ils à me répondre, si je leur disais que cette Junie est un personnage inventé, comme l'Emilie de Cinna, comme la Sabine d'Horace ? Mais j'ai à leur dire que, s'ils avaient bien lu l'histoire, ils auraient trouvé une Junia Calvina, de la famille d'Auguste, sœur de Silanus, à qui Claudius avait promis Octavie. (P 1, p. 255 ; cf. P 2, p. 259)

(7) Voir les préfaces de Britannicus et la liste des personnages de Bajazet et de Phèdre. Les citations des textes de Racine renvoient au Théâtre complét, éd. Jacques Morel et Alain Viala, Paris, Dunod, 1995 (Classiques Garnier ; la mise à jour de 1995 corrige la numérotation des vers de Britannicus, erronée à partir du vers 1060 dans les versions précédentes). La préface de 1670 sera désignée par " $P 1$ ", celle des éditions ultérieures par " I' 2 ".

(8) Dans sa biographie de Vespasien, Suétone mentionne "Iuniam Calzinam $e$ gente Augusti " (Vies des douze Césars, texte établi et traduit par Henri Ailloud, t. III, Paris, Les Belles Lettres, 1964, p. 66) ; cette mention permet à Racine de préciser que Junia Calvina "vécut jusqu'au règne de Vespasien " (P 2, p. 259). Le fait que cette précision ne figure que dans la seconde préface me semble indiquer que Racine a découvert la mention de Junia Calvina chez Suétone seulement après coup, en préparant Bérénice. 
Depuis l'abbé Dubos (9), la critique n'a cessé de minimiser le lien entre le personnage de Junie et son modèle historique; les raciniens semblent étonnamment unanimes à insister sur la totale liberté d'invention du poète, qui s'efforcerait de déguiser la "création apparemment gratuite de Junie » (10) par des justifications spécieuses destinées aux cornéliens (11). Mais affirmer que « Junia Calvina n'a rien à voir avec Junie " simplement parce qu' « elle n'a jamais été l'objet de l'amour de Néron " (12), c'est aller bien vite en besogne. De même, le fait que Racine, comme il le reconnaît lui-même, a "rectifi[é] les mœurs » de Junie en la représentant «plus retenue qu'elle n'était » $(P 1, p$. 255), n'abolit pas tout lien entre la création et son modèle. Pour mieux comprendre la «naissance » de Junie et pour en dégager la pleine signification, il faut rouvrir ce dossier trop rapidement fermé, et examiner de façon plus détaillée le rapport entre le personnage de Racine et la Junia Calvina de l'histoire. Si le dramaturge a choisi la

(9) Réflexions critiques sur la poësie et sur la peinture, 7* édition, Paris, Pissot, 1770, section XXIX (réimpr. Genève, Slatkine, 1967, pp. 71-72).

(10) Ronald W. Tobin, "Néron et Junie : fantasme et tragédie ", [in] Re-lectures raciniennes, éd. Richard L. Barnett, Paris-Seattle-Tübingen : PFSCL, 1986 (Biblio 17, vol. 16), p. 195 ; selon Tobin, Junie serait « un personnage que l'on ne retrouve ni chez. Tacite, ni chez Suétone " (p. 194).

(11) Cf. les jugements suivants qui illustrent cette trop rassurante unanimité : " le poète fait état d'une Junia Calvina - d'ailleurs peu convaincante, il faut l'avouer - qui est attestée dans un passage de l'A pocolokyntose " (Raymond Picard, La Carriere de Jean Racine, Paris, Gallimard, 1961, p. 150); " Junie ist, obzwar Racine sich bemiiht, ihre Identität mit einer Figur des Tacitus zu" bezeeisen (erstes und zaveites Vorwort), im Grunde eine Schöpfung Racines " (Jürgen von Stackelberg, "Tacitus und die Bühnendichtung der französischen Klassik", Germanisch-Romanische Monatsschrift 10 [1960], p. 399); “On ne peut pas prendre au sérieux ce que Racine dit, dans les préfaces de Britannicus, d'une jeune et belle Junia Calvina, mentionnée par Sénèque et Tacite, mais totalement étrangère à l'histoire de Britannicus " (Jacques Truchet, La Trage'die classique' en France, Paris, PUF, 1975, p. 78) ; "Despite his ingenious or disingenuous disclaimers, the character is a complete ineention "(John Campbell, Racine, "Britannicus ", London : Grant \& Cutler, 1990, p. 15). - Le jugement de Jacques Scherer est un peu plus nuancé : "Le personnage de Junie est entièrement inventé par Racine, tout au moins dans ses rapports avec Britannicus et avec Néron " (Racine et/ou la cérémonie, Paris, PUF, 1982, p. 176).

(12) Tobin, "Néron et Junie " , p. 195. 
sœur de Silanus pour donner une amoureuse à Britannicus et pour, en effet, " créer une femme idéale » (13), ce choix est tout sauf gratuit ; il s'avère, surtout, riche d'implications politiques qui nous intéresseront ici tout particulièrement.

Les " censeurs " de Junie, écrit Racine, la confondent avec Junia Silana, parce qu'ils n'ont pas " bien lu l'histoire ». C'est qu'ils n'ont lu qu'un manuel commode, l'Histoire romaine de Coëffeteau, où Junia Silana est nommée, alors que Junia Calvina y apparaît sans nom, en tant que sceur anonyme de Silanus (14).

Racine, lui, a très bien lu l'histoire, ne serait-ce que pour être paré contre les reproches des doctes et des cornéliens; Britannicus est la tragédie " que j'ai le plus travaillée", une pièce "solide", louée par les "connaisseurs » (P 2, p. 257), affirme Racine. Le dramaturge ne s'est pas contenté de Coëffeteau, il s'est renseigné de première main, chez les historiens et les poètes : non seulement Tacite, mais encore Suétone et Sénèque, AuluGelle et Ovide (15). Britannicus est «nourri » de Corneille (16), mais aussi imprégné de culture latine : la tragédie s'inscrit dans un réseau intertextuel complexe dont les Annales de Tacite ne constituent que le fil le plus manifeste.

Pour la création de Junie, c'est bien Tacite qui fournit les éléments déterminants, - non seulement parce que Racine était "alors si rempli de la lecture de cet excellent historien » (P 2, pp. 257-258), mais aussi pour des raisons

(13) Tobin, "Néron et Junie ", p. 209.

(14) J'ai consulté la deuxième édition de 1662 : Nicolas Coëffeteau, Histoire romaine, Lyon, Huguetan, 1662 ; sur Junia Silana : t. II, p. 16 ; sur Junia Calvina : t. 1, p. 692 ("Sa sceur avec laquelle il avoit esté calomnié de ne converser pas assez chastement, fut bannie d'Italie ").

(15) Voir, pour une étude intertextuelle destinée à élucider le symbolisme politique qui sous-tend la fuite de Junie dans le temple de Vesta, mon article : Junie, Auguste et le feu de Vesta : étude intertextuelle du dénouement de Britannicus ", PFSCL XXIII, 45 (1996), pp. 575-599.

(16) Voir Marie-Odile Sweetser, "Racine rival de Corneille : "innutrition" et innovations dans Britannicus », Romanic Reviezo 66 (1975), pp. 13-31. 
idéologiques, qui apparaîtront plus clairement à propos du rôle négatif joué dans cette affaire par Sénèque. Puisque Racine "renvoie [le lecteur] à cet auteur, qui aussi bien est entre les mains de tout le monde » (P 2, p. 258), cherchons donc dans Tacite les origines de Junie.

Pourvu que l'on ne borne pas la lecture des Annales aux seules pages traitant du meurtre de Britannicus (où le personnage ne figure point), on y trouve un certain nombre d'endroits qui nous informent de la tragique destinée de la famille de Junia Calvina. Cet arrière-plan historique s'avère indispensable à la compréhension, non seulement de certains vers de la pièce, mais de l'essence même du personnage de Junie.

Précisons tout d'abord (17) que Junia Calvina est la fille de Marcus Junius Silanus et d'Aemilia Lepida. Sa mère est, tout comme Agrippine, une arrière-petite-fille d'Auguste : comme Néron, Junia est donc descendante directe d'Auguste à la quatrième génération (par une filiation d'ailleurs exclusivement féminine : Julia Major $>$ Julia Minor > Aemilia Lepida). Le fait que Junie soit « de la famille d'Auguste » n'est pas seulement indiqué par Racine dans la préface, mais apparaît à plusieurs reprises dans le texte même de la pièce : ainsi, Burrhus souligne que la " nièce d'Auguste " (v. 244) est du "sang de César » (v. 241) ; Agrippine se réfère au "sang de mes aïeux qui

(17) Car la plupart des tableaux généalogiques reproduits notamment dans les éditions scolaires même les plus récentes sont tantôt muets, tantôt fautifs en ce qui concerne la famille de Junie ; ainsi, les "Classiques Bordas " (1994, p. 19) aussi bien que les "Classiques Larousse" (1991, pp. 16-17) indiquent Drusus (fils de Germanicus) comme père de Junie, ce qui ferait d'elle non pas la souur des Silani, mais la nièce d'Agrippine et de Caligula. (Ce Drusus était bien marié à une Aemilia Lepida, mais n'était pas pour autant le père de Junie.) Le nom du père est certes un détail négligeable, pourvu que l'on ne s'appuie pas sur de tels tableaux fautifs pour en tirer des conclusions comme celle-ci : en tombant amoureux de Junie, Néron "s'éprend de sa cousine, puisque tous deux sont les petits-enfants d'Agrippine I " (Alain Couprie, Racine, "Britannicus ", Paris, Nathan, 1992, pp. 84-85; mais on y apprend aussi que "Néron est par le sang le demi-frère de Britannicus "...). - Pour un tableau généalogique correct et assez complet, voir Georges May, "L'unité de sang chez. Racine" , RHLF 72 (1972), p. 214. 
brille dans Junie » (v. 228), Néron au "sang " dont elle sort (v. 623) ; Junie elle-même allègue les «Césars, auteurs de ma naissance » (v. 568).

Or, l'appartenance à la lignée d'Auguste fut pour Junia et ses frères moins un titre de gloire qu'une source de malheurs. Prétendants potentiels à l'empire, les Junii Silani causaient de l'ombrage à Agrippine et Néron et furent progressivement éliminés par eux. Le premier événement de la série appartient à la préhistoire explicite de Britannicus puisqu'il est retracé deux fois par l'Agrippine de Racine:

Par moi seule éloigné de l'hymen d'Octavie,

Le frère de Junie abandonna la vie,

Silanus, sur qui Claude avait jeté les yeux,

Et qui comptait Auguste au rang de ses aïeux. (vv. 63-66)

Silanus, qui l'aimait [= Octavie], s'en vit abandonné

Et marqua de son sang ce jour infortuné. (vv. 1141-1142)

Les vers de Racine correspondent, pour l'essentiel, au récit de Tacite (Annales XII, 3-9) (18) : après la mort de Messaline (épouse de l'empereur Claude et mère de Britannicus), l'ambitieuse Agrippine réussit à arranger deux mariages, son propre mariage incestueux avec son oncle Claude, ainsi que celui de son fils Domitius avec Octavie, fille de l'empereur. Ce double hymen amènera Claude à adopter Domitius, qui s'appellera dorénavant Néron et pourra ensuite « légitimement » (j'y reviendrai) succéder à son père adoptif, en écartant Britannicus. Agrippine « ne pouvait réaliser sans un crime » $(A n n . X I I, 3)$ ses projets : il fallut tout d'abord évincer Lucius Junius Silanus, à qui Claude avait déjà fiancé Octavie. Silanus fut donc accusé d'inceste avec sa sœur Junia Calvina; il « fut contraint de

(18) Toutes les citations des Annales proviennent de la traduction de Pierre Grimal, Paris, Gallimard (Folio), 1990 ; elles seront désignées dans le texte par l'abréviation Ann. 
se démettre de sa magistrature " $(A n n$. XII, 4) et se tua le jour même des noces d'Agrippine et de Claude. "Domitius, désormais fiancé et gendre, devient l'égal de Britannicus, grâce aux intrigues de sa mère " (Ann. XII, 9).

On aperçoit déjà comment Racine est parti de la réalité historique pour intégrer Junia Calvina dans l'action de sa pièce : l'histoire atteste les fiançailles entre Silanus et Octavie ; par analogie, le poète en " déduit " une alliance nouvelle entre la sœur de Silanus et le frère d'Octavie, entre Junie et Britannicus. Puisque le frère de Junia a été promis à la fille de l'empereur, quoi de plus "logique » que d'imaginer que Claude donne (19) en outre la sœur à son fils (vv. 558, 584), redoublant ainsi le lien entre les Silani et sa propre maison? L'invention du poète remplit simplement la «case " restée vide dans l'histoire. Se non è vero, è ben trovato.

L'exploitation créatrice de cet épisode va plus loin. Junia Calvina fut bannie d'Italie pour être rappelée plus tard par Néron, qui voulait ainsi manifester son «indulgence » après l'assassinat d'Agrippine (Ann. XIV, 12); elle n'était donc même pas à Rome au moment du meurtre de Britannicus. Mais, paradoxalement, cet exil fournit un autre point commun avec la Junie racinienne qui, elle aussi, est " absente de la cour " (v. 641) jusqu'au jour où Néron l'y fait amener de force. Racine se sert de la donnée historique, mais pour en modifier le sens et pour " rectifier les mœurs » du personnage : le bannissement temporaire, punition d'un (prétendu) inceste, se transforme en éloignement perpétuel, source de pureté et d'intégrité morale : " un cour [...] d'une infidèle cour ennemi dès l'enfance " (vv. 943-944). Du coup, l'acte de l'empereur change lui aussi de valeur morale : le rappel généreux de l'exil devient un rapt et un emprisonnement par lequel

(19) C'est cet accord paternel autorisant l'amour réciproque que Roland Barthes a désigné comme la "légalité " ou "légitimité " de l' "Eros sororal", sans y chercher un sens politique plus précis (Sur Racine', Paris, Seuil, 1963, p. 16). 
Néron, " devenu ravisseur, [...] de Silanus fait enlever la sœur » (v. 226).

Extrapolation par analogie et transformation méliorative, tels sont les deux procédés créateurs par lesquels Racine utilise ce premier élément de l'histoire de Junia Calvina rapporté par Tacite. Mais ce que Néron appelle, euphémiquement, " le malheur qui lui ravit son frère " (v. 412) ne resta pas le seul. Un autre frère (Marcus Junius Silanus) fut tué à l'instigation d'Agrippine, aussitôt après l'avènement de Néron : sa mort fut " la première du nouveau principat » $(A n n$. XIII, 1). L'explication de Tacite mérite d'être citée en entier:

Agrippine qui avait machiné le meurtre de son frère, L. Silanus, craignait de trouver en lui un vengeur, et le bruit courait, dans le peuple, qu'il fallait préférer à Néron, à peine sorti de l'enfance, et arrivé au pouvoir grâce à un crime, un homme d'âge mur, irréprochable, noble et, ce qui alors était un argument, l'un des descendants d'Auguste ; car Silanus, lui aussi [c'est-à-dire comme Néron], avait Auguste comme arrière-grand-père. Telle fut la cause de ce meurtre (ibid.) (20).

Tout en étant " paresseux " et dénué de grandes ambitions politiques, ce Silanus, par le simple fait de descendre d'Auguste, apparaissait donc comme un véritable prétendant au principat, à la fois plus légitime et plus populaire que Néron. Il fut empoisonné « au cours d'un repas » (ibid.) - ce qui préfigure de façon frappante l'empoisonnement de Britannicus.

Des années plus tard, peu avant l'incendie de Rome, le troisième frère de Junia (Decimus Junius Silanus Torqua-

(20) Le passage prouve qu'une filiation uniquement maternelle n'exclut pas les prétentions dynastiques : "Fïr die Weitergabe des julischen Erbes sind längst auch die Frauen als Zuischenglieder eingetreten l...l Ebenso erscheint $M$. Junius Silanus im Jahre 54 als möglicher Thronprätendent aus dem julischen Haus, obwohl er nur ïber drei Frauen : Julia d.Ä., Julia d.J. und Aemilia Lepida von Augustus abstammt " (Konrad Kraft, "Der politische Hintergrund von Senecas Apocolocyntosis " , Historia XV [1966], pp. 113-114). 
tus) fut « contraint de mourir, parce que, outre l'illustration de la famille des Junii, il se vantait d'avoir le dieu Auguste comme trisaïeul "; il s'ouvrit les veines pour prévenir la condamnation par Néron $(A n n . X V, 35)$.

Enfin, après la mort de Poppée, un neveu de Junia (Lucius Junius Silanus Torquatus), "que son éminente noblesse et les leçons de $C$. Cassius [...] avaient élevé à la hauteur de n'importe quelle dignité " (Ann. XV, 52), remarqué «par l'illustration de sa race et sa jeunesse, empreinte de modération " (Ann. XVI, 7), fut banni après avoir été faussement accusé d'inceste (encore...) avec sa tante Junia Lepida, la sœur de Junia Calvina. Emprisonné "dans un municipe d'Apulie nommé Bari », il "supportait, en sage, un sort tout à fait immérité », avant d'être tué par un centurion envoyé par Néron (Ann. XVI, 9). Pour que tout fût consommé, pour que même le nom des Junii Silani fût enseveli, il restait à le rayer du calendrier : sous prétexte qu'il ne fallait pas rappeler leurs «crimes ", le nom du mois de juin fut changé de Junius en Germanicus (Ann. XVI, 12).

Seule la mort du premier Silanus figure de façon explicite dans la tragédie de Racine ; mais c'est le sort de tous les Silani qui explique la véritable étendue de la " misère » (v. 296) et du " malheur » (v. 298, v. 614) de Junie (car elle est déjà malheureuse avant d'avoir perdu Britannicus). Sinon, comment pourrait-on justifier la façon dont elle se décrit elle-même dans son affrontement capital avec Néron : " une fille, / Seul reste du débris d'une illustre famille" (vv. 555-556) ; " une fille, / Qui vit presque en naissant éteindre sa famille » (vv. 611-612)? Modeste et prudente, Junie évite d'énumérer devant l'empereur tous ces forfaits; mais, fière et courageuse, elle n'omet pas de lui rappeler que son règne a été fatal, non seulement à un frère, mais à une famille entière. Dans ces vers synthétiques, Racine évoque donc toute l'histoire des Silani, en condensant des événements éparpillés dans le temps, et dont quelques-uns ne se sont même pas encore produits au moment où se joue la pièce. Dans Britannicus comme 
dans Mithridate, « le procédé de Racine est de concentrer, de construire un personnage et une époque en ramassant dans les quelques heures d'une action tragique, sans se soucier de la chronologie, des traits saillants recueillis sur un grand nombre d'années " (21). Infidèle à la chronologie, Racine reste fidèle au sens politique et moral de l'histoire telle que la transmet Tacite, dont le récit en apparence impassible trahit en fait une profonde indignation devant tant de crimes perpétrés contre des innocents.

Junie apparaît ainsi comme l'unique survivante d'une famille persécutée et exterminée par Agrippine et Néron pour des raisons proprement dynastiques; sans que les Silani se soient livrés à des activités révolutionnaires, leur appartenance à la lignée d'Auguste suffit pour que l'empereur se voie obligé de les liquider. Tout comme Britannicus, Junie est une victime politique de la prise du pouvoir par Néron, leur ennemi commun; celle-ci a frappé les deux amants pareillement, nécessitant « leur ruine " (v. 60) et causant « leur misère » (v. 296). Le statut d'orpheline malheureuse que Junie partage avec tant d'autres personnages raciniens - et l'analogie avec Aricie est particulièrement frappante (22) - est évidemment une source de pathétique, mais présente en même temps des aspects politiques.

Il me paraît impossible de réduire l'origine augustéenne de Junie à un détail extrinsèque et décoratif, ou encore à un simple prétexte commode pour justifier son enlèvement par Néron; cette origine fonde l'essence même du

(21) R.C. Knight, Racine et la Grèce, Paris, Nizet, 1974, p. 295 (1" éd. 1951).

(22) "J'ai perdu, dans la fleur de leur jeune saison, / Six frères... Quel espoir d'une illustre maison! " (Phèdre, vv. 423-424) ; "D'une tige coupable il [Thésée] craint un rejeton; / Il veut avec leur soeur ensevelir leur nom " (vv. 107-108). Aricie descend d'Erechthée, mythique fondateur d'Athènes ; Thésée ne doit l'accession au trône d'Athènes qu'à l'adoption de son père Egée par l'andion. - Il faudrait remonter jusqu'à Andromaque pour trouver l'origine de ces descendantes de " maisons" princières exterminées par des ennemis pour des raisons guerrières ou politiques: " J'ai vu trancher les jours de ma famille entière " (Andromaque, v. 929); cf. Astyanax lui-même, " reste de tant de rois sous Troie ensevelis" (v. 72). 
personnage, "de sa naissance, de sa vertu et de son malheur » (P 1, p. 257 ; P 2, p. 259). La naissance augustéenne explique non seulement le malheur qui s'abat sur sa famille, mais encore la vertu de Junie. Car loin d'être un idéal moral apolitique, sa "vertu, si nouvelle à la cour » (v. 417), cette "vertu conforme à son malheur » (v. 614), est conforme aussi à sa naissance : elle réincarne la vertu exemplaire d'Auguste, invoquée dans la première comme dans la dernière scène de la tragédie («vertus», v. 30 ; "vertu », v. 1738).

Auguste représente en effet, dans Britannicus, un point de référence absolu en tant que fondateur divinisé de l'empire, une origine plus mythique qu'historique de laquelle découle une légitimité à la fois politique et morale. Il est significatif que le texte racinien ne remonte pas aux sources du pouvoir d'Auguste lui-même et qu'il ne le rattache point à son père adoptif, "Divus Julius ", à qui il doit pourtant l'empire. Alors que l'histoire romaine après Auguste est amplement présentée, l'époque de la république et des guerres civiles est passée sous silence ; Jules César n'est même pas nommé, et les crimes d'Octave, si présents dans le Cinna de Corneille, sont expédiés par une très rapide allusion à la façon dont "Auguste a commencé » (v. 34). Dans la suite, la vertu d'Auguste ressort d'autant mieux qu'elle est explicitement opposée à la «tyrannie » de Caligula (v. 40). Au lieu de mettre à nu, comme le faisait Cinna, les origines violentes du pouvoir d'Auguste, Britannicus, adoptant la mythologie augustéenne, évoque ce règne comme un âge d'or révolu pour mieux pouvoir lui opposer la décadence qui l'a suivi.

Junie n'est pas le seul personnage de la pièce à descendre du fondateur de l'empire ; elle partage ce privilège, non pas avec Britannicus (nous y reviendrons), mais avec Agrippine ("Auguste votre aieul ", v. 84) et, par conséquent, avec Néron («Auguste, votre aïeul », v. 476). Mais il semble que, de tous ces descendants, elle seule soit restée fidèle à l'ancêtre et n'ait pas démérité de son héritage. Le texte la présente comme si elle descendait unique- 
ment d'Auguste, alors que chez Agrippine et Néron d'autres "humeurs " sont venues souiller le sang d'Auguste : "la fierté des Nérons " d'une part, " des fiers Domitius l'humeur triste et sauvage " d'autre part (vv. 3638). Il est d'ailleurs révélateur qu'Agrippine paraisse bien plus orgueilleuse d'être la fille de l'imperator Germanicus (vv. 156, 164, 844, 1172), incarnation de la maîtrise militaire, que de descendre de "Divus Augustus ", symbole de la vertu. Ainsi Junie, implorant la statue d'Auguste après la mort de Britannicus, peut même se présenter elle-même comme la dernière survivante de l'empereur : « Prince [...] protège en ce moment le reste de ta race » (vv. 1731-1732). Une des fonctions de l'" invention » de Junie, et certes pas la moindre, c'est d'introduire dans la pièce un personnage qui soit un représentant " pur » d'Auguste.

Corollaire de son origine augustéenne, la "vertu " de Junie ne correspond cependant pas à la vérité historique :

Cette Junie était jeune, belle, et, comme dit Sénèque : festivissima omnium puellarum. Elle aimait tendrement son frère, " et leurs ennemis, dit Tacite, les accusèrent tous deux d'inceste, quoiqu'ils ne fussent coupables que d'un peu d'indiscrétion ". Si je la représente plus retenue qu'elle n'était, je n'ai pas ouï dire qu'il nous fût défendu de rectifier les mours d'un personnage, surtout lorsqu'il n'est pas connu. (P 1, p. 255; cf. P 2, p. 259)

L'abbé Dubos a reproché à Racine la manière frauduleuse dont il utilise ici le texte de Sénèque :

Seneque, dans la Satyre ingénieuse qu'il écrivit sur la mort de l'Empereur Claudius, parle de Julia [sic]Calvina en homme qui la tenoit réellement coupable du crime d'inceste avec son propre frere, \& pour lequel elle avoit été exilée sous le regne de ce Prince. Racine rapporte une partie du passage de Seneque, d'une maniere à faire croire qu'il ne l'avoit pas lû tout entier (23).

(23) Réflexions..., p. 72. 
Effectivement, au lieu de rapporter l'accusation d'inceste dans les termes grivois de Sénèque (24), Racine la fait réfuter par Tacite en l'imputant aux « ennemis " de Silanus et Junia, c'est-à-dire à Agrippine et ses complices (cf. Ann. XII, 4).

Or, il paraît difficile de nier que Sénèque lui-même faisait partie de ces complices qui aidèrent Agrippine à préparer la prise du pouvoir par Néron. C'est précisément lorsque Junia Calvina fut bannie de Rome qu'Agrippine obtint que l'écrivain prestigieux fût rappelé de l'exil où l'avait envoyé Claude ; la mère de Néron voulait notamment " recourir à ses conseils pour réaliser leur espoir de prendre le pouvoir, étant donné que Sénèque, pensait-on, serait fidèle à Agrippine » (Ann. XII, 8 ; cf. Brit., vv. 11591166). Pendant un certain temps, ses espoirs ne furent pas déçus : l'Apocoloquintose, satire ménippée écrite peu après la mort de Claude pour tourner en dérision son apothéose, n'est pas seulement un acte de revanche personnelle de Sénèque sur celui qui l'avait banni ; cette " métamorphose en citrouille » (tel est le sens du titre) de l'empereur défunt est aussi une œuvre de propagande, destinée à glorifier et légitimer l'arrivée au pouvoir de Néron. En ridiculisant Claude, ce pamphlet vise à rabaisser les prétentions politiques de ses descendants, c'est-à-dire de Britannicus (25). Et si Sénèque répète l'accusation d'inceste contre Silanus, c'est parce que le fiancé d'Octavie - nous l'avons vu avait été le rival de Néron (26). En défendant, par l'intermédiaire de Tacite, Junia Calvina et son frère contre les

(24) "Car pour quelle cause, je vous prie, mit-il à mort son gendre Silanus? Parce quayant pour sceur la plus délicieuse fille du monde, qu'on appelait partout Vénus, il aima mieux l'appeler Junon " (Sénèque, L'Apocoloquintose 'tu dizin Claude', texte établi et traduit par René Waltz, Paris, Les Belles Lettres, 1966, p. 8).

(25) Voir l'article déjà cité de Konrad Kraft, "Der politische Hintergrund". - Néron n'était que le fils adoptif de Claude et pouvait se réclamer, en passant par Agrippine, directement d'Auguste : la dérision de Claude renforçait la position de Néron par rapport à Britannicus, qui ne descendait pas d'Auguste.

(26) Voir Kraft, " Der politische Hintergrund " , p. 116. 
accusations de Sénèque, Racine désavoue le propagandiste de Néron.

Il fait plus : il exclut Sénèque de sa pièce en lui préférant Burrhus.

"J'ai choisi Burrhus pour opposer un honnête homme à cette peste de cour [Narcisse] ; et je l'ai choisi plutôt que Sénèque. En voici la raison " (P 2, p. 258). Mais, à première vue, le passage qui suit ne donne pas vraiment de « raison » justifiant cette préférence pour Burrhus : citations de Tacite à l'appui, Racine semble louer les deux gouverneurs de Néron et ne formule aucun argument explicite contre Sénèque. Comme souvent dans ses préfaces, l'essentiel réside dans l'implicite et dans le non-dit : "Burrhus, après sa mort, fut extrêmement regretté à cause de sa vertu ». Et la mort et la vertu de Sénèque, alors? Racine n'en parle pas.

Lisons encore entre les lignes : Sénèque était "fameux", écrit Racine en citant Tacite (Ann. XIII, 2), « pour son éloquence et le tour agréable de son esprit, Seneca praeceptis eloquentiae et comitate honesta » (ibid.). Or, « le tour agréable de son esprit " est une traduction pour le moins étrange de " comitas honesta": cette expression désigne chez Tacite une " amabilité sans compromission" (trad. Grimal), " un agrément mêlé de noblesse ; [...] Sénèque sait plaire tout en restant dans l'honneur " (27). Dans la traduction tendancieuse de Racine, rien ne subsiste de cette qualité honorable - l'adjectif " honnête » est décerné à Burrhus. Comme l'a montré Roger Zuber (28), la seconde préface de Britannicus reprend en grande partie les tournures de Perrot d'Ablancourt : ainsi, la première phrase sur Bur-

(27) Alain Michel, Tacite et le destin de l'Empire, Paris, Arthaud, 1966, pp. 180-181 (c'est moi qui souligne).

(28) Roger Zuber, Le's "Belles Infidèles "et la formation du goût classique, Paris, Armand Colin, 1968, pp. 286-287. - En esquivant apparemment le problème de traduction posé par " comitas honesta" , d'Ablancourt écrit ceci : "Car les mours de Seneque estoient plus douces, \& celles de Burrhus plus seueres " (Les CEuvres de Tacite..., dernière édition, reveuë \& corrigée, Amsterdam, Elzevir, 1663, t. II, p. 108). Merci à Susanne Winter et à Erich Streitenberger pour ces renseignements. 
rhus et Sénèque ("Ils étaient tous deux... »), aussi bien que l'éloge de la vertu de Burrhus sont directement empruntés au traducteur des Annales. Mais pour opposer le caractère des deux gouverneurs de Néron, Racine préfère donner une traduction personnelle qui, dans le cas de Sénèque, est une «belle infidèle " : elle crée une image nettement moins vertueuse du philosophe que ne le fait la formule équilibrée de la " comitas honesta" .

"Le tour agréable de son esprit » : une expression semblable se trouve en fait chez Tacite ainsi que chez d'Ablancourt, mais dans un autre passage des Annales, qui traite de l'éloge funèbre de Claude - éloge prononcé par Néron, mais rédigé par Sénèque, premier " nègre » de l'empire (29). Personne ne put s'empêcher de rire en écoutant Néron louer la sagesse de Claude, "bien que le discours, composé par Sénèque, présentât beaucoup d'élégance, étant donné que ce personnage possédait un talent agréable [ingenium amoenum] et adapté aux oreilles de cette époque " $(A n n .$, XIII, 3) (30). Sénèque a écrit en même temps cet éloge hypocrite et (sous le couvert de l'anonymat) la satire contre Claude; parler du « tour agréable de son esprit " me paraît une façon euphémique de dénoncer son opportunisme qui l'amène à s'adapter à ce que le pouvoir attend de lui. Un siècle plus tard, les détracteurs de Sénèque n'ont pas manqué de l'entendre ainsi : "Racine, qui avait un tact si fin, un sentiment si exquis du beau moral, regardait Sénèque comme un charlatan » (31).

(29) "Les gens les plus âgés, qui peuvent, à loisir, comparer l'ancien temps et le présent, faisaient observer que Néron, le premier de tous ceux qui avaient eu le pouvoir, avait dû recourir à l'éloquence d'autrui " $(A m)$. XIII, 3). Cf. Aml. XIV, 16 ; et Britamicus, $v 120$ : "Sa réponse est dictée, et même son silence" .

(30) Perrot d'Ablancourt traduit ainsi : "Seneque, qui auoit l'esprit tre'sagreable \& tres-delicat ” (t. II, p. 109 ; je souligne).

(31) Cité par Diderot dans son Essai sur lés régne's de Clatude ét de' Néron, apologie passionnée du philosophe stoïcien contre ses détracteurs qui lui reprochent, entre autres, sa collaboration avec le tyran. Diderot ajoute: "Ce jugement valait bien la peine d'être appuyé d'une citation ", et propose d'autres raisons pour expliquer le fait que Racine préfère Burrhus à Sénèque : voir CEures, éd. Laurent Versini, t. 1, Paris, Laffont, 1994, p. 1097. 
Comment en effet le dramaturge aurait-il pu présenter, comme antithèse de Narcisse, un homme qui a lui-même accusé d'inceste Junie et justifié la spoliation de Britannicus? Sénèque a beau passer pour un précepteur rigoureux, un philosophe vertueux qui finira par abandonner Néron et mourir de façon glorieuse, il vaut mieux envoyer « loin de Rome » (v. 806) cet écrivain courtisan et vaniteux qui, lors de l'usurpation néronienne, s'est compromis en mettant son éloquence au service d'un pouvoir criminel. Ses nobles exhortations à la clémence, Racine les enlève à l'auteur du De clementia pour les attribuer à Burrhus (IV, 3) (32).

Si l'on accepte l'idée que Britannicus prend le contrepied de l'Apocoloquintose, on comprend peut-être mieux la portée politique des deux « inventions » grâce auxquelles la Junie racinienne se distingue de la Junia Calvina de l'histoire : pour démentir Sénèque, Racine doit donner une vertu sans faille à Junie et imaginer son alliance avec Britannicus afin de renforcer la légitimité de celui-ci. Dans la satire de Sénèque, qui cherche à présenter Néron, et non pas son prédécesseur, comme le véritable héritier d'Auguste, un discours de "Divus Augustus " lui-même, alléguant ses descendants assassinés, empêche la divinisation de Claude :

Le personnage que vous voyez, qui pendant tant d'années s'est caché sous mon nom, m'en a remercié en tuant deux Julies, mes petites-filles, l'une par le fer, l'autre par la faim, et un de mes arrière-petits-fils, L. Silanus [...] Et voilà le personnage dont vous voulez à présent faire un dieu? [...] vengez mes injures (33).

(32) Les rôles respectifs que jouent, dans la pièce elle-même, Burrhus et Sénéque (dont le nom est mentionné neuf fois) demanderaient un examen approfondi qui dépasse le cadre de la présente étude; il me paraît en tout cas nécessaire de tenir compte de la préface et de ne pas oublier que Sénèque apparaît ici comme l'auteur de l'Apocoloquintose.

(33) Apocoloquintose, éd. citée, pp. 10-11. 
La pièce de Racine non seulement rappelle à deux reprises que la mort de Silanus, que Sénèque impute ici à Claude, est en réalité l'œuvre d'Agrippine ; en imaginant en outre que ce soit précisément Junie - sœur de Silanus et descendante d'Auguste - qui refuse de reconnaître les droits de Néron pour rester fidèle au fils de Claude, Racine, tacitement, s'inscrit en faux contre la propagande néronienne et réhabilite Junie et Britannicus.

\section{Légitimités embrouillées (34) : Rome et la monarchie héréditaire}

Si Britannicus a besoin d'un tel soutien "augustéen » en la personne de Junie, c'est parce qu'il ne descend pas luimême d'Auguste : son illustre aïeul est en fait MarcAntoine, mari d'Octavie (la sœur d'Auguste), tandis que Néron, par sa mère, se rattache à Auguste en ligne directe. La différence généalogique peut sembler minime; elle est pourtant capitale sur le plan dynastique.

En effet, le père de Britannicus, bien que neveu de Tibère et frère de Germanicus, fut le premier princeps dénué de toute filiation directe (même adoptive) remontant à Auguste; il n'appartenait pas à la gens Julia au sens strict (35). Suétone précise que son grand-oncle Auguste, dans son testament, " ne l'institua pas héritier, si ce n'est en troisième ligne, presque comme un étranger » (36). Le successeur de Caligula doit l'empire moins à sa naissance

(34) "Premier décor de fond de Britanmicus, une tragédie dynastique. Racine a choisi la plus complexe, la plus embrouillée qui fut jamais " (Lucien Dubech, Jean Racine politiquc, Paris, Grasset, 1926, p. 70).

(35) "Clautius ans not himself in the direct line of de'scent from Augustus by birth or adoption, and he thus lacked the family names, Iulius and Cacsar. He aris the first Princeps in this position. I... I Clantius' son inherited from his mother as acell as his father the blood of Augustus" sister Octaria. But he faced, as potential rienls, direct descendants of the first Princeps. (Miriam T. Griffin, Nero : The End of a Dymasty, London, B.T. Batsford, 1984, p. 192). Cf. Kraft : "Je'tech eignete", anders als bei Clandius oder Britomnicus, bei Nero der Mutter Agrippina julisches Blut, und der Grofizater Germanicus a'ar durch die Adoption nach römischer Auffasiung cin e'chter Julier geivorden" (" Der politische Hintergrund ", p. 113).

(36) Dirus Claudius, IV (Vie's de's douze Césars, t. II, p. 115). 
qu'à la fortune, à l'armée et à l'argent. Dans une scène rocambolesque, Suétone raconte comment « à l'âge de cinquante ans il devint maître de l'empire, grâce au hasard le plus extraordinaire " : caché derrière un rideau, de peur d'être agressé par les assassins de Caligula, il fut découvert par un soldat, qui, " comme Claude, terrifié, se jetait à ses genoux, le salua empereur ". Claude, "promettant à chaque soldat quinze mille sesterces », fut proclamé empereur par l'armée, alors que le Sénat, faute d'héritier mâle d'Auguste, était sur le point de " changer le régime de l'Etat » et de rétablir la république (37).

Pour ne pas trop ternir l'image de Britannicus, Racine évite d'insister sur les défauts notoires de son père ; de même, il n'évoque sa mère Messaline qu'en passant, sans prononcer son nom ni mentionner les scandaleux adultères pour lesquels elle a été " condamnée » (v. 1123). Cependant, les allusions à la "simple obéissance » de Claude (v. 1244) et à " la cour de Claudius, en esclaves fertile » (v. 187), la présence de Narcisse qui est l'un de ces "esclaves", et le long récit d'Agrippine (IV, 2) sont là pour rappeler que ce personnage veule et grotesque, à en croire Tacite et Suétone, n'était certes pas né pour régner. Mais surtout, le texte de Racine reste scrupuleusement fidèle à la vérité historique en ce qu'il ne fonde jamais les prétentions de Britannicus sur un quelconque lien généalogique avec Auguste (avec l'exception notable du vers 1734, à laquelle je reviendrai). Britannicus est uniquement " le fils de Claudius » (v. 17, v. 837), le seul fils même qu'ait engendré « l'empereur son père » (v. 559) ; contre Néron, il n'avait que le soutien faible et infidèle des « amis de son père » (v. 1152, v. 323).

La légitimité dynastique de Britannicus est donc fondée non pas sur le rapport lointain avec l'aïeul, fondateur de l'empire, mais sur le rapport immédiat avec le père, précédent empereur. Dans une monarchie héréditaire, ces deux types de légitimité devraient coïncider : le père lui-même

(37) Divus Claudius, $X$ (Vies des douze Césars, t. II, pp. 118-119). 
n'aurait pu accéder au trône s'il ne descendait pas de l'aïeul qui se trouve à l'origine de la dynastie. Mais la situation à Rome est différente; nous verrons pourquoi. Or, est-ce que, dans la pièce de Racine, cette légitimité du fils de Claude suffit pour lui donner des droits supérieurs à ceux de Néron, qui est non seulement le fils adoptif de Claude, mais encore un descendant direct d'Auguste? Oui, selon Georges Couton : « Le fils de l'empereur défunt est, aux yeux des spectateurs du XVII* siècle surtout, qui interprètent toujours les situations historiques à l'aide des lois et des mours de leur temps, le détenteur d'une légitimité absolue. " (38) Il semble en effet évident que le texte dans son ensemble vise à présenter Britannicus comme le seul successeur légitime. Dès la première scène de la pièce, nous apprenons que c'est par les intrigues criminelles d'Agrippine, "déshéritant le fils de Claudius » (v. 17), que Britannicus "s'est vu précipiter [...] du trône, où le sang l'a dû faire monter " (vv. 61-62). Si l'on considère uniquement le critère de la naissance, Néron apparaît bel et bien comme un usurpateur : "l'heureux Domitius » a eu la chance d'être "nommé César », d'être "appelé de si loin à l'empire » (vv. 16-18), en dépit de tout ce que sa " naissance / Entre l'empire et [lui] avait mis de distance » (vv. 1119-1120).

Tel est également le point de vue de Coëffeteau :
Apres la mort de Claudius, la naissance \& la Iustice don- noient l'Empire à Britannicus, ieune Prince, dont les Romains auoient conçeus de grandes esperances, que la cruauté de Neron moissonna en leur fleur. D'un autre costé l'adoption que Claudius avoit fait [sic] de Neron, sembloit aussi luy en donner une iuste esperance. Et quoy que ce ne peust estre au preiudice de Britannicus [...] Neron foulant donc aux pieds le droit du sang, ravit l'Empire à Britannicus [...] (39).

(38) "Britnmnicus, tragédie des cabales", [in] Mélange's d'histoire littéraire" ( $x v^{\prime} I-x$ VII siécle) offerts a Raymond Lebè'gue', Paris, Nizet, 1969, p. 272.

(39) Histoire romaine, t. II, p. 1. 
Coëffeteau suit ici de très près Dion Cassius (Histoire romaine, LXI, 1), mais la dernière phrase semble de son propre cru : l'invocation du "droit du sang » se rattache en effet aux doctrines politiques de la France des Bourbons (40). Cependant, l'idéologie dynastique que cette formule exprime n'est point étrangère à la Rome impériale, où le " sang » compte beaucoup dans la succession au principat. Ainsi, la tirade dans laquelle Agrippine, furieuse du «mépris » que lui témoigne Néron, menace de rétablir « le fils de Claudius », en rappelant à l'armée la différence entre " le fils d'un empereur » et le «fils d'Ænobarbus " (vv. 837-848), n'est pas une invention anachronique de Racine, mais une imitation des Annales : l'on y entend Agrippine vociférer que Britannicus « était le véritable rejeton de Claude, digne de prendre en mains le pouvoir qui lui venait de son père, un pouvoir qu'exerçait un intrus, un fils adoptif » (Ann. XIII, 14). Tacite relate aussi que le testament de Claude "ne fut pas lu en public, pour éviter que la préférence donnée à un beau-fils sur un fils, injuste et mal acceptée, ne jette le trouble dans l'esprit de la foule » (Ann. XII, 69). Ces passages prouvent que la naissance était un facteur important dans le règlement de la succession, pour l'armée aussi bien que pour le peuple.

Un facteur important, mais pas le seul. Tout en invoquant le critère de la naissance selon lequel Britannicus est le seul successeur légitime de Claude, Racine a tenu également à présenter la position adverse qui justifie l'arrivée au pouvoir de Néron - position formulée par Burrhus, qui répond ainsi aux menaces d'Agrippine :

Madame, c'est un fils qui succède à son père.

En adoptant Néron, Claudius par son choix

De son fils et du vôtre a confondu les droits.

Rome l'a pu choisir. Ainsi, sans être injuste,

Elle choisit Tibère adopté par Auguste ;

(40) Cf. Robert Descimon et Alain Guéry, " Un Etat des temps modernes? ", Histoire de la France : l'Etat et les pouroirs, Paris, Seuil, 1989, Pp. 183-356 ; ici surtout pp. 230-231. 
Et le jeune Agrippa, de son sang descendu,

Se vit exclu du rang vainement prétendu. (vv. 860-866)

Si Racine prête ces mots à Burrhus, plutôt qu'à la " peste de cour "Narcisse, ce n'est pas pour discréditer cet « honnête homme ", mais pour souligner que ses arguments sont, $d^{\prime} u$ certain point de vue, tout à fait fondés. Pour ce soldat dévoué aux lois républicaines, la naissance ne peut donner aucun privilège à Britannicus, et l'on ne doit donc pas s'étonner de son "zèle sincère » (v. 859) pour le fils adoptif de Claude. Burrhus croit à la légitimité politique de Néron comme il croit encore à sa bonté morale, et dans les deux cas "Rome » semble, dans un premier temps, lui donner raison. Le gouverneur idéalise certes le " choix" de Rome, qui n'était pas aussi libre qu'il voudrait le croire ou le faire croire, et il devrait mieux connaître les crimes qui ont frayé le chemin à Néron : il refoule la vérité secrète de cette prise de pouvoir pour n'en garder que l'aspect public, officiel. Son argumentation juridique allègue précisément les structures et pratiques romaines qui ont permis à Agrippine de placer son fils sur le trône en maintenant une apparence de légalité.

En fait, la tirade de Burrhus donne une idée assez juste des modalités qui régissent réellement la transmission du pouvoir dans la Rome impériale : le princeps est désigné selon une combinaison hybride de critères héréditaires et électifs, où la naissance n'est qu'un facteur parmi d'autres; le testament de l'empereur décédé, le choix du Sénat et du " peuple ", celui de l'armée s'en mêlent sans que le résultat soit établi d'avance. De plus, le privilège de la naissance lui-même se trouve entamé par la pratique de l'adoption : non seulement Tibère, mais aussi Caligula (dont le père Germanicus avait été adopté par Tibère) doivent le principat à l'adoption. A Rome il n'y a donc point, comme il existe en France, une loi de succession formelle, uniquement fondée sur l'hérédité, par laquelle serait désigné sans équivoque, et ce dès le jour de sa naissance, un seul successeur absolument légitime : 
The chief problem presented by the method of succession was that there was no method to speak of. As the Principate was not an overt monarchy, there could be no acknowledgement of the hereditary principle, dominant as it was in practice and, a fortiori, no lazv of succession to regulate hereditary claims (41).

Si Burrhus présente une vision de jure de cet état de choses, les nombreuses références au rôle décisif joué par l'armée dans la désignation de l'empereur (vv. 769, 839$841,858,1169-1172,1185-1194,1256)$ donnent une image plus réaliste de ce qui détermine de facto le " choix » de «Rome». Tout cela, me semble-t-il, nous interdit d'affirmer que "Racine prête abusivement à l'empire romain le principe de l'hérédité de la couronne en usage dans la monarchie française " $(42)$; le principe de l'hérédité a une grande importance à Rome, mais il ne règle pas à lui seul la succession au principat. Et c'est bien ce que montre Racine.

On pourrait arrêter ici l'analyse et conclure que "Britannicus hésite entre deux conceptions de la succession » (43), entre le "droit du sang » et la légalité du "choix", entre une perspective "française » et une perspective "romaine ". Mais regardons de plus près : c'est Rome qui hésite ; c'est Britannicus qui expose cette hésitation, et ses consé-

(41) Griffin, Nero, p. 189.

(42) May, "L'unité de sang " , p. 227, à propos des " droits " de Junie (vv. 239-240). De même, Jacques Morel et Alain Viala écrivent, toujours à propos des droits de Junie : "Racine projette ici sur le monde romain des conceptions du XVIF" siècle " (Racine, Théâtre complet, p. 848); et Raymond Picard note à propos de ces vers : "Cet extrême souci des alliances du sang impérial, par lequel Burrhus justifie plus loin l'enlèvement de Junie, est plus louis-quatorzien que romain " (Racine, OEures completes, éd. Raymond Picard, t. I, Paris, Gallimard, 1950 [Bibl de la Pléiade], p. 1099). Pourtant, comme le montrent aussi bien la satire de Sénèque que les Annales de Tacite, descendre d'Auguste était réellement un " argument " dynastique ; et le souci des alliances du sang impérial existait également à Rome : ainsi, Tibère lui-même, "après s'être longuement demandé quels maris il donnerait à ses petites-filles ", propose des époux que le Sénat doit approuver (Ann. VI, 15).

(43) Laurence Lévy-Delpla, Racine, "Britannicus ", Paris, Hatier, 1988, p. 10. 
quences désastreuses pour l'Etat. Racine reconstitue, de façon assez fidèle, les différents facteurs qui déterminent la succession impériale, pour mettre à nu les défaillances du système romain qui font ainsi mieux ressortir les vertus de la monarchie héréditaire à la française. L'idéologie "louis-quatorzienne » se manifeste moins dans une description anachronique du régime politique de la Rome impériale que dans l'évaluation à laquelle ce régime se voit implicitement soumis. Car la tragédie non seulement "donne à voir qu'il n'est de bon prince que par droit de succession » (44), mais elle montre encore qu'il n'est de bonne monarchie que celle où la succession au trône est réglée par un critère indiscutable, tel celui de la primogéniture. "Ce n'est pas assez de dire que la Monarchie Royale et légitime est meilleure que la Démocratie ou Aristocratie, si on ne dit Monarchie dévolue par droit successif au mâle le plus proche du nom, et hors partage $[\ldots] »(45)$.

De ce point de vue, la tirade de Burrhus révèle un processus fatal : sous les apparences de la succession par filiation, un double "choix » (celui du père, l'adoption, d'abord ; celui de Rome ensuite) a subrepticement pris le pas sur la «naissance», justifiant l'éviction de Britannicus; au lieu d'être " né » empereur en vertu du sang du père, le successeur a été " nommé » par suite des intrigues d'une mère. Car le rôle d'Agrippine ne se réduit pas à celui d'une régente à qui est confié temporairement le pouvoir qui se transmet du père au fils : sa "voix " a littéralement "fait un empereur " (v. 158), « elle l'a couronné » (v. 1234), Néron lui « doi[t] l'empire »(v. 1223). C'est pour cela qu'elle se croit autorisée à demander une récompense et à exiger que son fils, simple "dépositaire " de son propre pouvoir (v. 1235), l'associe au règne; c'est pour cela que Néron, s'il veut régner seul, doit se montrer

(4) Alain Viala, Racinc' : la stratégic du cameilion, Paris, Seghers, 1990, p. 132.

(45) Jean Bodin, Le's Six liere's de' la République', ed. Gérard Mairet, Paris, le Livre de Poche, 199.3, p. 554. 
« ingrat » (passim) en tuant, pour commencer, Britannicus. Une logique économique, contractuelle (46), fondée sur l'équivalence entre les "bienfaits " et les "récompenses" (v. 1232), entre les " forfaits » et le " salaire " (v. 1196), pervertit en secret l'ordre politique : ce n'est pas le devoir public envers Rome, mais la dette individuelle envers sa mère qui détermine l'action du souverain.

Dans une perspective dynastique, l'adoption surtout est une pratique pernicieuse : elle brouille la distinction primordiale entre la famille impériale et les autres familles, elle " confond les droits » (v. 862) de l'héritier légitime et de l'usurpateur. A la suite de cette confusion, la légitimité elle-même, qui devrait être une valeur absolue, se trouve entraînée dans le monde contingent des cabales humaines par lesquelles une marâtre intrigante comme Agrippine réussit à placer sur le trône un fils qui " n'est point né pour l'empire » (v. 1468). Néron est vraiment un " parvenu de l'empire » (47) qui doit son ascension non à l'argent, mais à l'adoption (préparée en plus par un inceste) ; comme l'argent, l'adoption est un facteur de liberté individuelle et de mobilité sociale qui trouble la stabilité de l'ordre " naturel », généalogique (48).

Mais Britannicus précise aussi que le mal vient de plus loin, des origines de l'Etat; le fondateur de l'empire commit lui-même le péché originel :

Auguste, votre aïeul, soupirait pour Livie:

Par un double divorce ils s'unirent tous deux,

Et vous devez l'empire à ce divorce heureux. (vv. 476-478)

Certes, c'est Narcisse qui parle ici, pour inciter Néron à répudier Octavie ; mais, pour une fois, il dit la vérité.

(46) Cf. Catherine Spencer, La Tragédie du prince, Paris-Seattle-Tübingen, I'FSCL, 1987 (Biblio 17, vol. 32), pp. 364-367.

(47) Raymond Picard, Présentation de Britannicus, [in] Racine, OEuvrés complètes, p. 373.

(48) Cf. Philip Lewis, "L'adoption dans le théâtre de Racine ", xvIr siècle, 46 (1994), pp. 773-785. 
Auguste avait répudié Scribonia - qui venait pourtant de faire ses preuves en donnant naissance à une fille (Julia, l'arrière-grand-mère de Junia Calvina) - pour pouvoir épouser Livie, pourtant déjà mariée et enceinte. Auguste, "séduit par sa beauté, l'enlève à son mari - on ne sait si ce fut malgré elle - et cela avec une telle hâte que, sans même lui laisser le temps d'arriver au terme de sa grossesse, il l'introduit à son foyer alors qu'elle est enceinte. Par la suite, elle n'eut plus aucune progéniture " $(A n n . \mathrm{V}, 1)$. Faute de fils, Auguste dut alors recourir à l'adoption pour désigner un successeur ; ainsi, le premier fils de Livie, Tibère, « que l'hymen plaça dans sa famille » (v. 479), fut "adopté par Auguste » (v. 864) et accéda finalement à l'empire, aux dépens du petit-fils d'Auguste, du " jeune Agrippa, de son sang descendu " (v. 865). Cet Agrippa fut d'abord exilé par Auguste, que Livie " avait si bien subjugué » $(A n n . \mathrm{I}, 3)$, puis assassiné immédiatement après l'avènement de Tibère. "Le premier acte notable du nouveau principat fut le meurtre de Postumus Agrippa " (Ann. I, 6), comme la mort violente de M. Junius Silanus sera " la première du nouveau principat " de Néron : à Rome comme en Turquie, le nouveau souverain ne doit pas trop tarder à éliminer ceux qui sont "sortis d'un sang/Qui les a de trop près approchés de son rang " (Bajazet, vv. 107-108).

Auguste créa un fatal précédent : de façon remarquablement analogue, Claude, succombant aux caresses d'Agrippine, adopta Néron et lui ouvrit ainsi le chemin au trône, au détriment de Britannicus. Racine exploite cette analogie entre l'intronisation de Tibère et celle de Néron en contaminant les passages relatifs de Tacite : la manoeuvre d'Agrippine à la mort de Claude, par laquelle le peuple " apprit en même temps votre règne et sa mort » (v. 1194), fut déjà celle de l'ambitieuse Livie, "d'une violence toute féminine " $(A n n . \mathrm{I}, 4)$, qui avait tout arrangé pour que " l'on apprît en même temps qu'Auguste était mort et que Nero [=Tibère] prenait le pouvoir $»(A n n . \mathrm{I}, 5)$. 
Au moment du divorce, Auguste s'appelait encore Octave ; le divorce est la seule action d'Octave mentionnée explicitement dans Britannicus, action en apparence "domestique " qui ne ternit pas beaucoup l'image brillante des vertus de l'empereur. Mais dans le contexte de la pièce, ce divorce - qui affecte non pas une famille quelconque, mais la " maison " impériale - apparaît en fait moins comme un scandale privé que comme une faute politique, la seule que l'empereur semble avoir commise : s'il était resté fidèle à Scribonia après la naissance du premier enfant, il aurait sans doute réussi à procréer des héritiers mâles pour fonder une véritable dynastie. Le divorce, en revanche, a brisé l'unité naturelle de la gens Julia en lui associant, par le moyen arbitraire de l'adoption, une lignée étrangère, la gens Claudia ; désormais, le sang d'Auguste sera mêlé à « la fierté des Nérons » (v. 38), mélange personnifié par Caligula, Agrippine et Néron.

Le divorce d'Auguste n'est pas un acte isolé ou fortuit ; il est solidaire de ce défaut structurel que même les historiens modernes continuent d'identifier comme un problème fondamental du nouveau régime établi par Auguste :

Il y a, dans cette machine bien montée, quelque chose qui manque de précision, qui est abandonné, dans une large mesure, au jeu des circonstances; et cette indécision touche, malheureusement, un point vital du régime : Auguste n'a pas voulu ou n'a pas su fixer une règle de succession au pouvoir, d'accès au principat. [...] L'Empire romain a toujours gravement souffert de cette faiblesse congénitale (49).

Selon l'idéologie dynastique, fondée sur une mystique du sang, Auguste n'a failli que sur ce point : ayant conquis et mérité l'autorité monarchique grâce à sa «vertu » personnelle, il aurait dû veiller à ce que cette autorité reste, au-delà de sa propre mort, liée à son sang, qui est censé perpétuer sa vertu. Il a bien fait d'imposer à

(49) Eugène Albertini, L'Empire romain, 4" éd., Paris, JUF, 1970, p. 38. 
Rome une succession quasi héréditaire pour garantir que le pouvoir ne quitte pas sa famille, mais il n'est pas allé assez loin dans cette entreprise, qui aurait requis la proclamation ouverte de la monarchie.

S'il y a un " message " politique dans Britannicus, c'est ici, à mon avis, qu'il faut le chercher : le drame politique $y$ prend la forme d'une " tragédie des cabales" , non pas pour insinuer que les cabales font " partie de l'essence même de la monarchie, comme il [Racine] la voyait et la comprenait » (50), mais pour les présenter comme la conséquence inéluctable d'une certaine forme de monarchie défectueuse, mal constituée. L'absence d'une véritable loi de succession, fondée sur des principes invariables et indiscutables, suscite les intrigues les plus criminelles de la part des ambitieux qui veulent conquérir et conserver le pouvoir souverain. En revanche, si la primogéniture seule décidait de l'accession au principat, des mancuvres comme celles d'Agrippine seraient déjouées d'avance parce qu'elles n'auraient aucune chance d'aboutir. De par ses lois fondamentales, la monarchie héréditaire des Bourbons peut se vanter d'être supérieure à la Rome impériale : «Point de brigues, point de cabales dans un Etat pour se faire un roi : la nature en a fait un. (51) »

Si Britannicus ne contient point de débat explicite sur les avantages respectifs de la monarchie et de la république, tel qu'il se trouve dans Cinna (II, 1), il me semble en revanche possible d'y déceler une confrontation implicite de deux formes de monarchie, l'une française, l'autre

(50) Couton, "Britamicus, tragédie des cabales", p. 277. Cf. Annie Ubersfeld, "Racine" ", [in] Mamul d'histeire littirnire de la France, éd. Abraham et Desné, Paris, Editions Sociales, 1966, pp. 298-299.

(51) Bossuet, Politique tirée de's propure's parole's de l'Ecriture sainte, livre II ; cité d'après: Politique de Bossue't, éd. Jacques Truchet, Paris, Colin, 1966, p. 106. Une telle préférence pour la monarchie héréditaire est d'ailleurs exactement contraire au point de vue de Tacite lui-mème, qui semble plaider pour l'abandon complet du principe de l'hérédité (cf. Michel, Tacit', p. 168). Racine reprend l'analyse tacitéenne de la décadence de lempire romain, mais, conformément au contexte idéologique de la France des Bourbons, il en arrivè des conclusions opposées. 
romaine. Sur le plan «constitutionnel », l'empire romain peint par Racine est un régime ambigu, indécis : une monarchie qui n'ose pas dire son nom, une autocratie de fait camouflée par des façades républicaines. L'illusion tragique de Burrhus, c'est justement de croire qu'entre république et principat existe un compromis qui permettrait qu'en même temps "Rome soit toujours libre, et César tout-puissant » (v. 214). Sa situation en porte à faux, entre ses convictions républicaines et sa loyauté à l'empereur, reflète ce compromis boiteux entre république et monarchie que constitue l'Etat lui-même. L'ambiguïté fondamentale du régime éclate à chaque crise de succession, lorsque s'entremêlent le " choix " de Rome et le principe héréditaire (52). La constitution de l'empire est restée en retard sur la réalité historique : depuis Auguste, l'exercice du pouvoir est monarchique, l'autorité du prince absolue ; mais l'accession au principat, la transmission de ce pouvoir absolu n'est pas contrôlée selon des principes monarchiques, par une " loi fondamentale » purement et rigoureusement dynastique. La tragédie de Racine semble suggérer que non seulement la lutte meurtrière pour le pouvoir, mais encore la dérive vers la tyrannie sont le résultat inévitable d'une telle constitution hybride, "monstrueuse ", qui favorise les cabales et institutionnalise l'usurpation; les vices de Néron ne peuvent se répandre sur Rome qu'à la faveur d'un « vice de construction » de l'État lui-même.

\section{Politique du couple}

Ainsi, le fils unique de l'empereur voit ses droits contestés au nom des lois et traditions romaines elles-mêmes,

(52) Cf. Jochen Bleicken, Verfassungs- und Sozialgeschichte des Römischen Kaiserreiches, t. I, Paderborn, Schöningh, 1989, p. 112: "Das Nachfolgeproblem des römischen Kaisertums ist ein Spiegelbild seiner doppelten Grundlage. Die beiden sich widerstreitenden Prinzipien [i.e. principe juridique, électif vs. principe dynastique] treten bei beinahe jeder Nachfolge zutage und stören einander. " 
exprimées par Burrhus. La légitimité de Britannicus devrait être absolue et incontestable, mais ne l'est pas; toute fondée sur le rapport avec le père, il lui manque ce lien de consanguinité avec l'aïeul que possède Néron. Pour assurer la légitimité de Britannicus, pour élever le "droit du sang » au-dessus de ce brouillamini de brigues et de cabales, Racine lui adjoint Junie : la descendante d'Auguste est censée apporter une caution supérieure, quasi éternelle, au fils de Claude. C'est seulement à travers elle que Britannicus peut se réclamer d'Auguste; c'est dans la brève tirade nécrologique de Junie que « le dernier représentant du sang des Claudii » (Ann. XIII, 17) devient, à titre posthume seulement, un « neveu ", un petit-fils d'Auguste :

Rome, dans ton palais, vient de voir immoler

Le seul de tes neveux qui te pût ressembler (vv. 1733-1734).

Au cours de la pièce, il était déjà apparu que Britannicus, à moins d'être "fortifi[é] du parti de Junie " (v. 1252), n'aurait aucune chance de reconquérir le trône; leur union représente le véritable danger pour Néron :

Vous savez que les droits qu'elle [Junie] porte avec elle

Peuvent de son époux faire un prince rebelle (vv. 239-240);

Grâces aux dieux, Seigneur, Junie entre vos mains

Vous assure aujourd'hui le reste des Romains (vv. 373-374).

Par son origine, Junie devient un pion sur l'échiquier dynastique : pour des raisons stratégiques, son mariage avec Britannicus, qui avait jadis été arrangé par Claude (vv. 557-558), est maintenant favorisé par Agrippine et contrecarré par Narcisse. Une telle politique matrimoniale est toute romaine : si Claude avait "jeté les yeux " (v. 65) sur Silanus pour le marier à Octavie, c'était déjà pour se rattacher davantage à Auguste et pour neutraliser les prétentions des Silani (53). Suétone signale par ailleurs que 
Claude lui-même avait d'abord été fiancé à Aemilia Lepida (la future mère de Junia Calvina), avant de la répudier " parce que ses parents avaient offensé Auguste » (54).

Sur le plan de l'intrigue, de la "trame politique" analysée par Georges Couton (55), l'alliance entre Britannicus et Junie n'aboutira pas; dans la réalité de la lutte pour le pouvoir, les deux amants se trouvent embarqués dans une coalition par trop hétérogène avec Agrippine, leur ancienne ennemie, dont le soutien intéressé est d'autant plus nuisible qu'elle-même le croit décisif. Mais au-delà des calculs stratégiques et des rapports de force réels, le couple Britannicus-Junie symbolise, sur le plan des valeurs, une véritable utopie politique, que son échec tragique ne saurait que renforcer : leur alliance réunirait les deux «branches » de la dynastie julio-claudienne, la gens Julia et la gens Claudia. L'union entre la " nièce d'Auguste » et le «fils de Claudius » rétablirait à Rome une légitimité entière, joignant celle fondée sur le père à celle qui découle de l'ancêtre.

Ce n'est donc pas un personnage à lui seul, mais un couple qui détient la pleine légitimité ; non pas un homme seul, mais un homme et une femme. Il faut souligner ici l'importance politique accordée à la femme : si Junie ne peut certes pas elle-même prétendre à l'exercice du pouvoir (et Agrippine, "maîtresse " de Rome, est là pour illustrer les fantasmes misogynes de la "femme au pouvoir » (56)), Racine lui confère néanmoins un rôle capital, celui d'incarner la légitimité et la vertu sans lesquelles l'autorité de l'homme ne saurait être complète. La loi salique est à la fois respectée et contournée.

L'amour partagé de Junie et Britannicus se trouve ainsi doublé d'une légitimité dynastique également partagée. Le couple d'amants présente à la fois une idylle amoureu-

(54) Divus Claudius, XXVI (Vies des douze Césars, t. II, p. 135).

(55) Voir son article déjà cité sur "Britannicus, tragédie des cabales" .

(56) Cf. Pierre Ronzeaud, "La femme au pouvoir ou le monde à l'envers" , xvIr. siècle 27 (1975), pp. 9-33. 
se et un idéal politique : il figure le véritable couple impérial digne de régner, souverain potentiel dont l'avènement constitue le telos latent, le dénouement rêvé mais irréalisable de la tragédie : si les deux amants parvenaient à se tirer des intrigues pour présider au destin de Rome, ils destitueraient l'autre couple de la tragédie, cette mésalliance monstrueuse et précaire formée par le " couple maudit » (57) Agrippine et Néron. Et, une fois mariés, les époux ne manqueraient certes pas d'assurer la continuité de la dynastie (58), alors que sous Néron « l'empire vainement demande un héritier » (v. 473). Néron en effet mourra sans fils; avec lui s'éteindra la lignée des empereurs julio-claudiens : "Galba, le successeur de Néron, n'avait aucun degré de parenté avec la famille des Césars " (59). La suite de l'histoire est connue : quelques années avant Britannicus, Corneille l'avait mise en scène dans Othon.

Mais Racine lui-même a écrit la suite d'Othon : c'est Bérénice. Après les guerres civiles et l'année des quatre empereurs consécutive à la mort de Néron, Rome semble retrouver de nouveau la stabilité et aboutir, avec la dynastie des Flaviens, à un véritable système de succession héréditaire. A Vespasien a succédé, comme il se doit, son fils aîné Titus ; la passation des pouvoirs s'est déroulée parfaitement (60) : l'empereur jouit d'une légitimité entière, que l'alliance avec une reine orientale ne pourrait que diminuer. Ici, le couple n'a pas de raison d'être politique : Titus doit sacrifier son amour et régner seul. Alors que Britannicus montre les désastres qui mettent fin à la lignée

(57) Ingrid Heyndels, Le Conflit racinien, Bruxelles, Editions de l'Université de Bruxelles, 1985, p. 47.

(58) A la différence des couples cornéliens, dont Marc Fumaroli a mis en évidence la "vocation fondatrice " ("Du Cid à Polyeucte : une dramaturgie du couple" , [in] Héros et orateurs, Genève, Droz, 1990, p. 410), le couple racinien a pour tâche non de fonder la Cité, mais de continuer la monarchie, d'assurer la perpétuité de la dynastie.

(59) Suétone, Galba, Il (Vie's de's douze Césars, t. III, p. 3).

(60) Voir Christian Delmas, "Bérćnice et les rites de succession royale " , xill siécle 39 (1987), pp. 395-401. 
des Julio-Claudiens, Bérénice met en scène les sacrifices nécessaires à la fondation d'une nouvelle dynastie.

Discrètement mais sûrement (pourvu que l'on y fasse attention), le texte racinien construit ainsi une certaine perspective politique capable d'enrichir notre perception du couple amoureux. Pour dégager cette perspective, je me suis appuyé presque exclusivement sur les éléments du texte se référant à la généalogie des personnages et à l'arrière-plan historique qui explique la situation présente. S'agissant d'une pièce si manifestement dominée par les considérations dynastiques, une telle expédition ardue et périlleuse dans le labyrinthe généalogique, pour en développer l'embarras incertain, m'a paru légitime, et même nécessaire (61). Cette importance de la généalogie sur le plan politique fait d'ailleurs pendant au poids de l'hérédité chez Racine, souvent mis en valeur par la critique d'inspiration psychologique ou «biologique » .

Il va de soi, d'autre part, que cette analyse du couple amoureux est partielle, dans la mesure où elle laisse de côté des dimensions essentielles du personnage de théâtre, telles les passions qui l'animent et les actions qu'il entreprend. La place manque ici pour entreprendre une étude détaillée de ces aspects, mais je voudrais du moins, pour terminer, en esquisser quelques rudiments.

Rappeler le rôle politique du couple amoureux ne revient pas à considérer l'amour comme un simple prétexte, subordonné aux intérêts politiques. Jean-Pierre Miquel a pu écrire : "L'amour de Junie et Britannicus ? probable et même certain, mais né d'une alliance à caractère politique - l'amour est venu après, pour conforter un projet commun : la reconquête du pouvoir » (62). Une telle interprétation me paraît aussi réductrice que celle qui ne voit

(61) On peut parfaitement étendre à Britannicus cette observation de Georges May : " une pleine connaissance de la doublure politique de l'intrigue de Phèdre a pour condition nécessaire une idée précise de la généalogie des personnages" ("L'unité de sang", p. 228).

(62) "Une mise en scène de Britannicus", Cahiers de l'Association Internationale des Etudes françaises, 31 (1979), p. 153. 
en Junie qu'une amoureuse apolitique ; au lieu de dissocier ces deux aspects ou d'établir une hiérarchie entre eux, il vaut mieux reconnaître leur fusion. "Drame politique et drame privé sont inséparables " (63) ; dans le cas de Britannicus et de Junie, politique et amour vont ensemble.

Les deux amants ne s'aiment donc pas de cet " amour politique », si fréquent dans l'œuvre de Corneille (64), qui n'est que le masque, le moyen ou le résultat de l'ambition et de l'amour des grandeurs. Mais la présence d'un enjeu politique pose en effet la question du rôle joué par l'ambition : cette " passion ambiguë " (65) risque de ternir la pureté de l'amour, qui pourrait alors apparaître comme un sentiment intéressé. Par conséquent, Racine doit diminuer le rôle de l'ambition au profit de l'amour : c'est bien cette dernière passion qui semble, subjectivement, dominer chez Britannicus et Junie. Nécessité analogue sur le plan de l'action dramatique : pour que leur vertu ne soit pas compromise, les amoureux doivent être tenus un peu à l'écart de " tous ces complots " (v. 1253) que trament les autres personnages (66).

L'amour sert ici moins à reléguer l'ambition politique au second plan qu'à la purifier en la dépouillant de ses composantes d'amour-propre. Car il faut souligner que, chez Britannicus, l'amour ne fait pas complètement disparaître l'ambition ; Racine cherche à établir un équilibre entre les deux passions, ce qui entraîne une certaine ambiguïté quant aux "véritables » motivations du personnage.

(63) Picard, Présentation de Britannicus, p. 374. - J'avoue ne pas comprendre la position de Jacques Scherer : " à s'en tenir exclusivement au plan politique, Britannicus a tort d'aimer Junie et Junie a tort de refuser Néron. I_a contradiction est partout." (Racine' et/ou la cérímonic', p. 103). Ce " plan politique "serait-il "exclusivement " celui de l'amour-propre et des intérêts égoïstes?

(64) Voir Octave Nadal, Le' Sentiment de l'amour dans l'a'utere de Pierre' Corn'ille', Paris, Callimard, 1948, pp. 243-253.

(65) Jacques Morel, Racinc, p. 61.

(66) Cf. Marie-Rose Carré, "Lè problème de l'action chez les personnages vertueux dans le théâtre de Racine ", l'Esprit créateur. VIII (1968), pp. 89101. 
Le fils de Claude semble s'intéresser « surtout » (vv. 351, 517) à son amour; mais en même temps, il ne s'est pas encore résigné à son statut d'héritier spolié, et l'enlèvement de Junie lui donne enfin l'occasion de rassembler les adversaires de Néron pour reconquérir non seulement son amante, mais encore le trône perdu (vv. 319-322, 347350). Face à Agrippine, il prend soin de cacher son jeu (I, 3 ; III, 5), car il voudrait s'« engager sous son nom plus loin qu'elle ne veut " (v. 358) - c'est-à-dire détrôner Néron. Son perfide confident pourra s'appuyer sur ces déclarations pour justifier l'assassinat de Britannicus : “il aspirait plus loin qu'à l'hymen de Junie » (v. 1663). Dans sa confrontation avec l'empereur (III, 8), Britannicus n'omet pas de braver “Domitius » (v. 1040) en lui reprochant son usurpation et sa tyrannie, au lieu de se montrer conciliant pour obtenir la libération de Junie. Mais il finit par renoncer à combattre pour ses droits; tout en rappelant qu'il a été "banni du rang de [ses] aïeux » et que Néron se pare "de leur dépouille », il déclare se contenter de Junie, que l'empereur feint de lui céder, et avoue qu'il " lui laisse le reste [= l'empire!] avec moins de regret " (vv. 1489-94). Le rappel emphatique de l'usurpation indique cependant qu'il ne s'agit pas ici d'un simple abandon des intérêts politiques au profit du bonheur privé, mais plutôt d'un sacrifice généreux offert à l'être aimé : par émulation, Britannicus imite le geste désintéressé de Junie elle-même qui lui a "sacrifié l'empire et l'empereur » (v. 1498).

Ce sacrifice constitue en fait la véritable action politique de Junie : si elle repousse avec " persévérance " (vv. 418, 958) l'invitation de Néron à " passer du côté de l'empire » (v. 588), ce n'est pas seulement par amour, mais encore parce qu'elle connaît les crimes auxquels il doit cet empire et qu'elle refuse de légitimer l'usurpation. Elle ne montre ni ambition personnelle ni soif de vengeance; mais elle ne manque pas de défendre les droits de Britannicus (vv. 643648), tout comme ceux d'Octavie (vv. 630-632). " En res- 
tant fidèle à Britannicus, Junie reste fidèle à tout ce qu'il y a de légitime à Rome " (67).

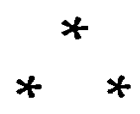

Concluons. Opposée aux cabales de la cour, l'idylle du couple amoureux ne se situe pourtant pas en dehors des valeurs de la politique, mais constitue elle-même l'un des pôles du monde politique tel que le présente Racine. A amputer le couple amoureux de cette dimension, on risque de prendre Britannicus pour " une tragédie machiavélique, et même machiavéliste " (68). L'on pourra certes se moquer de ces amants trop parfaits, trop purs, trop pitoyables, et préférer la fascination bien plus grande qu'exercent sur nous les passions troubles et les machinations diaboliques d'Agrippine et de Néron. Il n'en reste pas moins que le couple formé par Britannicus et Junie s'avère un élément indispensable de la structure dramatique et idéologique de la tragédie : face à l'usurpation fondée sur l'amour-propre et la haine, il représente la légitimité dynastique alliée à l'amour réciproque.

Volker SCHRÖDER

(67) Judd D. Hubert, Essai d'exégèse racinienne : le's secrets témoins, Paris, Nizet, 1956, p. 112. Cf. Harriet Stone, "Authority and Authorship : Néron's Racine", [in] Re-lecture's raciniennes, p. 167 : "Junie never falters in her dedication to patriarchal law'. I... I Junie represents a power that is both political and moral, a power that reconciles public need and personal desire" ". - Loin de représenter une fuite hors du politique, la " retraite " de Junie chez les Vestales corrobore en fait cette analyse (voir note 15 ).

(68) P'hilip Butler, Classicisme et baroque dans l'auvere de Racine, Paris, Nizet, 1959, p. 178. 